Wechselkursschwankungen,

Außenhandel und Arbeitsmärkte

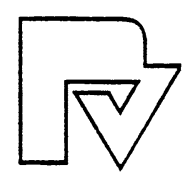




\section{Wirtschaftswissenschaftliche Beiträge}

\section{Informationen über die Bände 1-110 sendet Ihnen auf Anfrage gerne der Verlag.}

Band 111: G. Georgi, Job Shop Scheduling in der Produktion, 1995. ISBN 3-7908-0833-4

Band 112: V. Kaltefleiter, Die Entwicklungshilfe der Europäischen Union, 1995.

ISBN 3-7908-0838-5

Band 113: B. Wieland, Telekommunikation und vertikale Integration, 1995.

ISBN 3-7908-0849-0

Band 114: D. Lucke, Monetäre Strategien zur Stabilisierung der Weltwirtschaft, 1995. ISBN 3-7908-0856-3

Band 115: F. Merz, DAX-Future-Arbitrage, 1995. ISBN 3-7908-0859-8

Band 116: T. Köpke, Die Optionsbewertung an der Deutschen Terminbörse, 1995. ISBN 3-7908-0870-9

Band 117: F. Heinemann, Rationalisierbare Erwartungen, 1995. ISBN 3-7908-0888-1

Band 118: J. Windsperger, Transaktionskostenansatz der Entstehung der Unternehmensorganisation, 1996. ISBN 3-7908-0891-1

Band 119: M. Carlberg, Deutsche Vereinigung, Kapitalbildung und Beschäftigung, 1996. ISBN 3-7908-0896-2

Band 120: U. Rolf, Fiskalpolitik in der Europäischen Währungsunion, 1996. ISBN 3-7908-0898-9

Band 121: M. Pfaffermayr, Direktinvestitionen im Ausland, 1996. ISBN 3-7908-0908-X

Band 122: A. Lindner, Ausbildungsinvestitionen in einfachen gesamtwirtschaftlichen Modellen, 1996. ISBN 3-7908-0912-8

Band 123: H. Behrendt, Wirkungsanalyse von Technologie- und Gründerzentren in Westdeutschland, 1996. ISBN 3-7908-0918-7

Band 124: R. Neck (Hrsg.) Wirtschaftswissenschaftliche Forschung für die neunziger Jahre, 1996. ISBN 3-7908-0919-5

Band 125: G. Bol, G. Nakhaeizadeh/

K.-H. Vollmer (Hrsg.) Finanzmarktanalyse und -prognose mit innovativen quantitativen Verfahren, 1996. ISBN 3-7908-0925-X

Band 126: R. Eisenberger, Ein Kapitalmarktmodell unter Ambiguität, 1996. ISBN 3-7908-0937-3

Band 127: M. J. Theurillat, Der Schweizer Aktienmarkt, 1996. ISBN 3-7908-0941-1

Band 128: T. Lauer, Die Dynamik von Konsumgütermärkten, 1996. ISBN 3-7908-0948-9
Band 129: M. Wendel, Spieler oder Spekulanten, 1996. ISBN 3-7908-0950-0

Band 130: R. Olliges, Abbildung von Diffusionsprozessen, 1996. ISBN 3-7908-0954-3

Band 131: B. Wilmes, Deutschland und Japan im globalen Wettbewerb, 1996. ISBN 3-7908-0961-6

Band 132: A. Sell, Finanzwirtschaftliche Aspekte der Inflation, 1997. ISBN 3-7908-0973-X

Band 133: M. Streich, Internationale Werbeplanung, 1997. ISBN-3-7908-0980-2

Band 134: K. Edel, K.-A. Schäffer, W. Stier (Hrsg.) Analyse saisonaler Zeitreihen, 1997.

ISBN 3-7908-0981-0

Band 135: B. Heer, Umwelt, Bevölkerungsdruck und Wirtschaftswachstum in den Entwicklungsländern, 1997. ISBN 3-7908-0987-X

Band 136: Th. Christiaans, Learning by Doing in offenen Volkswirtschaften, 1997.

ISBN 3-7908-0990-X

Band 137: A. Wagener, Internationaler Steuerwettbewerb mit Kapitalsteuern, 1997.

ISBN 3-7908-0993-4

Band 138: P. Zweifel et al., Elektrizitätstarife und Stromverbrauch im Haushalt, 1997.

ISBN 3-7908-0994-2

Band 139: M. Wildi, Schätzung, Diagnose und Prognose nicht-linearer SETAR-Modelle, 1997. ISBN 3-7908-1006-1

Band 140: M. Braun, Bid-Ask-Spreads von Aktienoptionen, 1997. ISBN 3-7908-1008-8

Band 141: M. Snelting, Übergangsgerechtigkeit beim Abbau von Steuervergünstigungen und Subventionen, 1997. ISBN 3-7908-1013-4

Band 142: Ph. C. Rother, Geldnachfragetheoretische Implikationen der Europäischen Währungsunion, 1997. ISBN 3-7908-1014-2

Band 143: E. Steurer, Ökonometrische Methoden und maschinelle Lernverfahren zur Wechselkursprognose, 1997. ISBN 3-7908-1016-9

Band 144: A. Groebel, Strukturelle Entwicklungsmuster in Markt- und Planwirtschaften, 1997. ISBN 3-7908-1017-7

Band 145: Th. Trauth, Innovation und Außenhandel, 1997. ISBN 3-7908-1019-3 


\section{Ansgar Belke}

\section{Wechselkursschwankungen, Außenhandel und Arbeitsmärkte}

Neue theoretische und empirische Analysen im Lichte der Europäischen Währungsunion

Mit 42 Abbildungen und 63 Tabellen

Springer-Verlag Berlin Heidelberg GmbH 


\title{
Reihenherausgeber
}

Werner A. Müller

\author{
Autor \\ Professor Dr. Ansgar Belke \\ Universität Wien \\ Fakultät für Wirtschaftswissenschaften \\ und Informatik \\ Hohenstaufengasse 9 \\ 1010 Wien \\ Österreich \\ E-mail: ansgar.belke@univie.ac.at
}
Als Habilitationsschrift auf Empfehlung der wirtschaftswissenschaftlichen Fakultät Bochum gedruckt mit Unterstützung der Deutschen Forschungs- gemeinschaft

ISSN 1431-2034

Die Deutsche Bibliothek - CIP-Einheitsaufnahme

Belke, Ansgar: Wechselkursschwankungen, Außenhandel udn Arbeitsmärkte: neue theoretische und empirische Analysen im Lichte der Europäischen Währungsunion / Ansgar Belke. - Heidelberg: Physica-Verl., 2001

(Wirtschaftswissenschaftliche Beiträge; Bd. 183)

$$
\text { ISBN 978-3-7908-1386-9 ISBN 978-3-642-57614-0 (eBook) }
$$

DOI 10.1007/978-3-642-57614-0

Dieses Werk ist urheberrechtlich geschützt. Die dadurch begründeten Rechte, insbesondere die der Übersetzung, des Nachdrucks, des Vortrags, der Entnahme von Abbildungen und Tabellen, der Funksendung, der Mikroverfilmung oder der Vervielfältigung auf anderen Wegen und der Speicherung in Datenverarbeitungsanlagen, bleiben, auch bei nur auszugsweiser Verwertung, vorbehalten. Eine Vervielfältigung dieses Werkes oder von Teilen dieses Werkes ist auch im Einzelfall nur in den Grenzen der gesetzlichen Bestimmungen des Urheberrechtsgesetzes der Bundesrepublik Deutschland vom 9. September 1965 in der jeweils geltenden Fassung zulässig. Sie ist grundsätzlich vergütungspflichtig. Zuwiderhandlungen unterliegen den Strafbestimmungen des Urheberrechtsgesetzes.

\footnotetext{
(C) Springer-Verlag Berlin Heidelberg 2001

Ursprünglich erschienen bei Physica-Verlag Heidelberg 2001
}

Die Wiedergabe von Gebrauchsnamen, Handelsnamen, Warenbezeichnungen usw. in diesem Werk berechtigt auch ohne besondere Kennzeichnung nicht zu der Annahme, dass solche Namen im Sinne der Warenzeichenund Markenschutz-Gesetzgebung als frei zu betrachten wären und daher von jedermann benutzt werden dürften. Umschlaggestaltung: Erich Kirchner, Heidelberg

SPIN $10797853 \quad 88 / 2202-543210$ - Gedruckt auf säurefreiem und alterungsbeständigem Papier 


\section{Vorwort}

Die vorliegende Studie befasst sich aus theoretischer und empirischer Perspektive mit dem Zusammenhang von Wechselkursänderungen, Außenhandel und Beschäftigung. Die Untersuchung bezieht sich auf die realwirtschaftlichen Effekte der Europäischen Währungsunion. Die Idee und Konzeption der vorliegenden Arbeit wurden im wesentlichen im Rahmen meiner Forschungsaufenthalte am Center for Economic Research (CentER), Tilburg/Niederlande, und am Centre for European Policy Studies (CEPS), Brüssel, geboren und weiter entwickelt. Prägend für den Blickwinkel der Arbeit waren die wissenschaftliche Zusammenarbeit einerseits mit Wim Kösters, einem Mitinitiator des Manifests von deutschen Hochschullehrern der Volkswirtschaftslehre gegen eine zu frühe Einführung des Euro sowie andererseits mit Daniel Gros, dem Mitverfasser der klassischen Studie ,Ein Markt, eine Währung'. Letztere strich bereits Anfang der neunziger Jahre realwirtschaftliche Vorteile einer relativ raschen Einführung des Euro für eine gewisse Auswahl an europäischen Volkswirtschaften heraus. Die weitere Motivation ergab sich angesichts der Tatsache, dass die faktische Einführung des Euro als Gemeinschaftswährung am zum 1. Januar 1999 genau in die Zeit der Anfertigung der Arbeit fiel, fast wie von selbst. Ähnlich stimulierend wirkten die schon damals heftigen und bis heute nicht verstummenden Diskussionen um die Vor- und Nachteile einer Europäischen Währungsunion und die Ursachen der anhaltend hohen Arbeitslosigkeit.

Einzelne Teile der Arbeit wurden auf internationalen Konferenzen vorgestellt und profitierten (hoffentlich) von zahlreichen kritischen Anmerkungen der Zuhörer. Zu den Tagungen zählten unter anderem der 'Annual European Economic Association Congress', das 'Annual Econometric Society European Meeting', die 'European Association of Labor Economists Annual Conference', das 'European Public Choice Society Annual Meeting', die 'International Conference of the Society for Computational Economics' und insbesondere das 'International Seminar on Macroeconomics (EEA und NBER)' in Gerzensee/Schweiz. Darüber hinaus wurden Inhalte der Studie auf der internationalen Konferenz, The Open Economy Macromodel - Past, Present and Future' an den Universitäten Bar Ilan und Ben Gurion (beide Israel) sowie auf dem Workshop 'Designing EU-US Monetary Relations' des 'Institute for International Economics' (IIE) in Washington/DC präsentiert. Gleichzeitig stießen Bestandteile der Argumentation auch in der Politik auf Interesse, wie beispielsweise im Rahmen eines Hearings im Ausschuß für 
Wirtschaft, Währung und Industriepolitik des Europa-Parlamentes in Brüssel zum Thema 'Adjustment Mechanisms in the Case of Asymmetric Shocks'.

Die Studie wurde gedruckt mit Unterstützung der Deutschen Forschungsgemeinschaft, der ich deshalb zu Dank verpflichtet bin. Für kritische Kommentare danke ich Daniel Gros, meinem akademischen Betreuer Wim Kösters sowie Dieter Bender und Manfred Lösch. Großer Dank gebührt Matthias Göcke für die enge und fruchtbare Zusammenarbeit bei der Entwicklung eines theoretischen Instrumentarium zur Analyse der Beschäftigungseffekte von Wechselkursunsicherheit bei Zeitpfadabhängigkeiten auf Arbeitsmärkten sowie Martina Kamp für die enge Forschungskooperation im Bereich ,EWU und Arbeitsmarktreformen'. Martin Hebler danke ich für kritische Hinweise zu den Teilen 3 und 6 der vorliegenden Arbeit. Dank gebührt darüber hinaus Ulrich Reuter, Christian Einhaus sowie Sonja Kisselmann und für ihre unermüdliche und sorgfältige redaktionelle Unterstützung bei der Erstellung der Endfassung der Arbeit. Schließlich und vor allem danke ich meiner Frau Claudia für Ihre Geduld und Unterstützung in den letzten Jahren.

Ansgar Belke

Wien, November 2000 


\section{Inhaltsverzeichnis}

Seite

Abkürzungsverzeichnis XV

1. Einleitung

2. Nominale Rigiditäten und der Beitrag flexibler Wechselkurse $\mathrm{zu}$ Arbeitsmarktanpassungen in offenen Volkswirtschaften

2.1 Motivation des Problems

2.2 Ein einfaches Basismodell: Darstellung und numerische Simulation.

2.3 Können die EWS-Krisen 1992/93 als ein 'kontrolliertes' Experiment bezeichnet werden?

2.4 Realer wiedervereinigungsbedingter Aufwertungsbedarf der DM: Gelang Italien und Großbritannien die Anpassung tatsächlich schneller als Frankreich?

2.4.1 Operationalisierung der Reihen und Spezifikation der Tests

2.4.2 Empirische Überprüfung durch strukturbrucherweiterte ADF-Tests.

2.4.3 Beurteilung der Schätzergebnisse

2.5 Invariable Wechselkurse:

Beschäftigungspolitische Kosten oder Nutzen der EWU?.......

2.5.1 Wie sinnvoll ist der Einsatz der Wechselkurspolitik zur Kompensation länderspezifischer Schocks?

2.5.2 Verstärkt die EWU die durch nominale Rigiditäten bedingten realwirtschaftlichen Effekte von Schocks?...

2.5.3 Beeinflußt die EWU das Ausmaß nominaler Rigiditäten? 56

2.6 Fehlende Wechselkursflexibilität:

ein starkes Argument gegendie EWU? 
3. Zur Politischen Ökonomie der Situation auf EU-Arbeitsmärkten zum Start der EWU:

Mancur Olson versus Insider-Outsider-Theorie......

3.1 Problemstellung.

3.2 Zur Ausgangslage: Ist die Arbeitslosigkeit in Westeuropa eine Folge des Verhaltens von Interessengruppen im polit-ökonomischen Kräftefeld?

3.2.1 Die Olson-Hypothesen .................................................. 78

3.2.2 Die Insider-Outsider-Theorie _....................................... 83

3.2.3 Arbeitslosigkeit als Folge polit-ökonomischer Anreize. 84

3.3 Gemeinsamkeiten und Unterschiede des Olson-Ansatzes und der Insider-Outsider-Theorie

3.3.1 Gemeinsamkeiten..................................................... 87

3.3.2 Unterschiede ............................................................ 96

3.3.3 Zur empirischen Validität beider Ansätze ..................... 99

3.4 Implikationen für die Beschäftigungspolitik und für Prognosen der Beschäftigungsentwicklung in der Europäischen

Währungsunion

3.5 Implikationen für eine Politische Ökonomie der Beschäftigungspolitik

3.5.1 Strategische Implementierungshemmnisse geeigneter Politiken

3.5.2 Zeitpfadabhängigkeit der Beschäftigungspolitik

3.5.3 Strategische Optionen einer geeigneten Beschäftigungspolitik.

3.5.3.1 Timing-Aspekte

3.5.3.2 Reform-Design

3.5.3.3 Ideologie-Aspekte 125

3.6 Zusammenfassung und Ausblick 
4. EWU, Geldpolitik und Reform der europäischen Arbeitsmärkte: Ein neues Modell zur währungspolitischen Endogenisierung von Rigiditäten

4.1 Einführung.

4.2 Die EWU als ein Olson-Schock für sklerotisierte Arbeitsmärkte?

4.2.1 Separierende Analyse der EWU und der europäischen Arbeitslosigkeit

4.2.2 Inhaltliche Zusammenführung von Teilaspekten als Forschungsziel

4.2.2.1 Theoretische und empirische Eckpunkte für eine Synthese

4.2.2.2 Die positive Ökonomik dynamischer Inkonsistenz

4.2.2.3 Die positive Ökonomik rigider Arbeitsmärkte..

4.3 Ein neues Basismodell............................................................... 140

4.4 Modellergebnisse................................................................ 145

4.4.1 Glaubwürdige Verpflichtung auf eine Geldmengenregel als Referenzfall

4.4.2 Diskretionäre Geldpolitik: Dynamische Inkonsistenz .. optimaler Politiken

4.4.3 Graphische Darstellung der Modellergebnisse

4.5 Vergleich der Gesamtwohlfahrt unterschiedlicher geldpolitischer Regimes ........................................................................... 166

4.6 Zusammenfassung und Interpretation der Ergebnisse.............. 174

4.6.1 Reformnachteil der EWU?.......................................... 174

4.6.2 Reformvorteil der EWU? ........................................... 177

4.6.3 Geldlohnrigidität und Arbeitsmarktreformen ................ 181

4.6.4 Abfederung von Strukturreformen durch die Geldpolitik? 183

4.6.5 Die Bedeutung der Persistenz der Arbeitslosigkeit........ 184

4.6.6 EWU: verstärkte Anreize zur Koordinierung von Reformen?.......................................................... 185

4.7 Schlußbemerkungen und Ausblick........................................ 187 
5. Der Einfluß externer Schocks auf EU-Arbeitsmärkte

5.1 Motivation der Untersuchung:

Warum sollte die Bedeutung externer Schocks

erstmals gesondert analysiert werden?

5.2 Die Theorie optimaler Währungsräume im Lichte der empirischen Literatur

5.3 Ein neuer direkter Test der Theorie optimaler Währungsräume 203

5.3.1 Arbeitslosigkeit 204

5.3.2 Beschäftigung in der verarbeitenden Industrie............. 214

5.4 Robustheit der Testergebnisse 220

5.4.1 Robustheit I: Der Einfluß des Wechselkurses. 220

5.4.2 Robustheit II: Der Einfluß der Fiskalpolitik 226

5.4.3 Robustheit III: Der Einfluß der Geldpolitik 229

5.4.4 Robustheit IV: Der simultane Einfluß potentieller 'Shock Absorber'

5.5 Interpretation der Ergebnisse 243

5.6 Schlußfolgerungen und Implikationen für die EWU-Debatte.. 251

6. Der Einfluß der Wechselkursunsicherheit auf EU-Arbeitsmärkte .. 256

6.1 Entwurf der Untersuchung: Welche Anhaltspunkte bietet die Literatur zum Einfluß der Wechselkursunsicherheit auf den Außenhandel?

6.1.1 Theorie 266

6.1.2 Empirische Studien 270

6.1.2.1 Messung des Risikos. 270

6.1.2.2 Resultate 279

6.1.2.3 Schlußfolgerungen

6.2 Motivation der Untersuchung:

Warum sollte die Bedeutung der Wechselkursunsicherheit für Arbeitsmärkte gesondert analysiert werden?

6.3 Investitionen in die Beschäftigung bei Unsicherheit:

Der 'Optionswert des Wartens' 
6.3.1 Das Basismodell: keine Risikoaversion........................ 327

6.3.2 Das Modell bei Risikoaversion ...................................... 332

6.4 Der 'Optionswert des Wartens' und der 'Wert der

Optionsschaffung'

6.5 Empirische Evidenz für Westdeutschland.............................. 347

6.5.1 Hat die Wechselkursvolatilität einen signifikanten Einfluß auf den deutschen Arbeitsmarkt?................................ 347

6.5.1.1 Spezifikation und Schätzergebnisse.................. 348

6.5.1.2 Beurteilung der Schätzergebnisse .................... 358

6.5.2 Wechselkursvolatilität und deutscher Arbeitsmarkt:

Eine Neubetrachtung unter Berücksichtigung von

'Common Long-Term Trends' und Strukturbrüchen......

6.5.2.1 Ökonometrische Spezifikation und Zeitreiheneigenschaften der Daten ................................. 372

6.5.2.2 Ein Fehlerkorrekturmodell ohne Strukturbrüche 381

6.5.2.3 Brüche in der Langfristbeziehung zwischen der Wechselkursvolatilität und der Beschäftigung. 384

6.5.2.4 Zwischenfazit ............................................... 392

6.6 Empirische Evidenz für EU-Volkswirtschaften ...................... 393

6.6.1 Eine vorläufige Arbeitsdefinition der

Wechselkursunsicherheit ........................................... 395

6.6.2 Einfache Tests: erste Resultate .................................... 409

6.6.2.1 Arbeitslosigkeit .............................................. 423

6.6.2.2 Beschäftigung in der verarbeitenden Industrie.. 426

6.6.2.3 Investitionen............................................... 427

6.6.3 Robustheit der Testergebnisse ..................................... 433

6.6.3.1 Robustheit I: Der Einfluß potentieller 'Shock

Absorber': Realer Wechselkurs, Geldpolitik und \$-Wechselkurs-Variabilität............................... 433

6.6.3.2 Robustheit II: Symmetrische Schocks ............. 445

6.6.3.3 Robustheit III: Simultanität der Regressoren und das 'Missing Variable'-Problem ....................... 453

6.7 Schlußfolgerungen und Implikationen für die EWU-Debatte.. 468 
7. Ein Modell für Hysteresis in der Beschäftigung bei

Wechselkursunsicherheit - eine neue Sicht

7.1 Einführung ....................................................................... 483

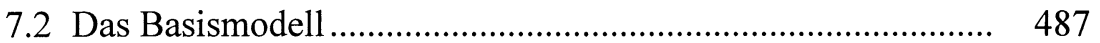

7.3 Entscheidung unter Sicherheit ................................................. 491

7.3.1 In der Vorperiode beschäftigt (aktiv) ............................. 491

7.3.2 In der Vorperiode nicht beschäftigt (passiv)................. 492

7.3.3 Breite des Hysteresis-Bandes .......................................... 493

7.4 Das Modell bei einmaliger Wechselkursunsicherheit und der Möglichkeit des Wartens

7.4.1 Die Implikationen von Wechselkursunsicherheit und der Optionswert des Wartens ................................. 494

7.4.2. In der Vorperiode beschäftigt (aktiv) ............................. 496

7.4.2.1 Kein Wiedereintritt ......................................... 496

7.4.2.2 Wiedereintritt ................................................. 500

7.4.3 In der Vorperiode nicht beschäftigt (passiv) ................. 504

7.4.3.1 Kein Wiederaustritt......................................... 504

7.4.3.2 Wiederaustritt................................................ 509

7.4.4 Breite des Hysteresis-Bandes bei Wechselkursunsicherheit

7.5 Das Modell bei zwei aufeinanderfolgenden stochastischen

Wechselkursänderungen.................................................... 514

7.5.1 In der Vorperiode beschäftigt (aktiv) ........................... 514

7.5.2 In der Vorperiode nicht beschäftigt (passiv) ................. 516

7.6 Numerische Beispiele........................................................... 519

7.7 Zusammenfassung und Schlußfolgerungen............................. 522

8. Ein neuer theoretischer Ansatz zur Aggregation:

Mikro- und Makro-Hysteresis in der Beschäftigung bei

Wechselkursunsicherheit

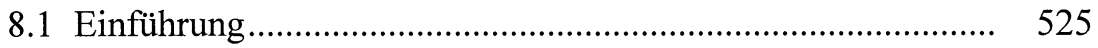

8.2 Das Makro-Modell bei Wechselkurssicherheit ....................... 528 
8.3 Das Makro-Modell bei Wechselkursunsicherheit ................... 536

8.4 Zwischenfazit und Implikationen für die EWU-Debatte.......... 543

8.5 Neue Algorithmen für Tests der Arbeitsmarkteffekte der Wechselkursvariabilität auf der Basis eines nichtlinearen theoretischen Aggregationsansatzes ................... 544

8.5.1 Einleitende Bemerkungen ............................................ 545

8.5.2 Eine lineare Approximation der Unsicherheitseinflüsse auf die Beschäftigung:

Die Modellierung von 'Play'-Hysteresis........................ 546

8.5.3 Ein Algorithmus zur Erfassung des linearen 'Play' ........ 548

8.5.4 Die Eigenschaften des Regressionsmodells.................. 560

8.5.5. Ein Beispiel einer empirischen Anwendung .................. 562

8.6 Schlußfolgerungen................................................................ 572

9. Wechselkursunsicherheit und der deutsche Arbeitsmarkt:

Eine Kointegrationsanwendung des Autoregressiven

Distributed-Lag-Ansatzes....................................................... 574

9.1 Einführung .................................................................... 574

9.2 Das Modell: Wie beeinflußt die Wechselkursunsicherheit die Arbeitsmärkte? ................................................................ 578

9.3 Tests der Existenz von Langfristbeziehungen .......................... 582

9.3.1 Kointegrationstests:

Der Pesaran, Shin und Smith-ARDL-Ansatz................ 585

9.3.1.1 Theoretische Fundierung................................. 585

9.3.1.2 Anwendung auf westdeutsche Arbeitsmarktdaten........................................................ 588

9.3.2 Einheitswurzeltests der einzelnen Zeitreihen................ 596

9.3.3 Kointegrationstests: Der Johansen-Systemansatz.......... 598

9.3.3.1 Kointegrationstests in Modellen mit der Wechselkursvolatilität als exogenem I(0)-Regressor ..... 601

9.3.3.2 Kointegrationstests in Modellen mit der Wechselkursvolatilität als exogener I(1)-Variable ......... 605 
9.4 Eine Anwendung des ARDL-Ansatzes auf die

Kointegrationsanalyse ....................................................... 612

9.4.1 Theoretische Fundierung............................................ 612

9.4.1.1 Schätzung von Langfristkoeffizienten ............. 612

9.4.1.2 Schätzung der Koeffizienten des Fehlerkorrekturmodells......................................................... 616

9.4.2 Anwendung auf westdeutsche Arbeitsmarktdaten........ 619

9.4.2.1 Schätzung der Ordnung der

Distributed-Lag-Funktion ............................... 619

9.4.2.2 Schätzung der Langfristbeziehungen ............... 619

9.4.2.3 Schätzung der resultierenden

Fehlerkorrekturmodelle .................................. 626

9.5 Dynamische Prognosen der Wachstumsrate der westdeutschen Beschäftigung auf der Basis von Variablen für eine offene Volkswirtschaft ......................................................... 635

9.6 Schlußfolgerungen und Implikationen für die Debatte um die Arbeitsmarktwirkungen der Wechselkursunsicherheit............. 640

10. Zusammenfassung und Ausblick ................................................ 644

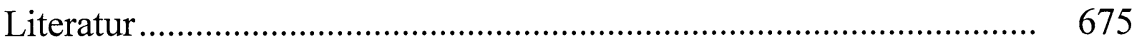

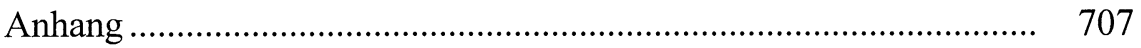

Tabellen- und Abbildungsverzeichnis................................................ 757 


\section{Abkürzungsverzeichnis}

Abb. : Abbildung

AIC : Akaike-Informationskriterium

ARCH : "autoregressive conditional heteroscedasticity"

ARDL : "autoregressive distributed lag"

Aufl. : Auflage

ausgen.: ausgenommen

Bd. : Band

BSP : Bruttosozialprodukt

BIP : Bruttoinlandsprodukt

Bd. : Band

BLUE : "best linear unbiased estimator"

CentER: CentER for Economic Research, Tilburg (Niederlande)

CEPR : Center for Economic Policy Research, London

CEPS : Centre for European Policy Studies, Brüssel

c.p. : ceteris paribus

dens. : denselben

ders. : derselbe

dies. : dieselbe oder dieselben

Diff : Differential

DIW : Deutsches Institut für Wirtschaftsforschung

DGP : datengenerierender Proze $\beta$

DSP : differenzstationärer Prozeß

Erg. : Ergebnis

ERM : "European Exchange Rate Mechanism"

EWS : Europäisches Währungssystem

EWU : Europäische Währungsunion

EZB : Europäische Zentralbank 
f. : folgende

Fed : Federal Reserve System

ff. : fortfolgende

FGLS : "feasible generalized least squares"

GARCH: "generalized autoregressive conditional heteroscedasticity" GBR : Großbritannien

gdl : "general distributed lag"

GIVE : "generalized instrumental variables estimator"

GLS : "generalized least squares"

H1 : Hypothese $1(\mathrm{H} 2=$ Hypothese 2 usw. $)$

Hrsg. : Herausgeber

i.i.d. : "independent and identically distributed"

IMF : Internationaler Währungsfonds

IOT : Insider-Outsider-Theorie (der Arbeitslosigkeit)

IV : Instrumentvariable (-nverfahren)

IVE : "instrumental variables estimator"

i.V.m. : in Verbindung mit

Jg. : Jahrgang

Kap. : Kapitel

KQ... : Kleinste Quadrate...

log. : logarithmiert

LR... : Likelihood Ratio...

Mass. : Massachusetts

MDS : "martingale difference sequence"

ML... : "maximum likelihood"...

NAIRU: "non-accelerating inflation rate of unemployment"

NAWRU: "non-accelerating wage inflation rate of unemployment"

NBER : National Bureau of Economic Research

NLS : "nonlinear least squares" 
o.ä. : oder ähnliches

OCA : 'optimum currency area'

OECD : Organisation for Economic Co-Operation and Development

OLS : "ordinary least squares"

PDL : "polynomial distributed lag"

PPP : "purchasing power parity", Kaufkraftparität

q.e.d. : quod erat demonstrandum

$\mathrm{RE}$ : rationale Erwartungen

RLS : "recursive least squares"

RWI : Rheinisch-Westfälisches Institut für Wirtschaftsforschung

S. : Seite

$\mathrm{SCH} \quad$ : Schwarz-Informationskriterium

SUR : "seemingly unrelated regression"

Tab. : Tabelle

TSLS : "two-stage least squares"

3SLS : "three-stage least squares"

TSP : trendstationärer Proze $ß$

v.a. : vor allem

VAR : "vector autoregressive" oder Vektorautoregression

Vol. : "volume"

WiSt : Wirtschaftswissenschaftliches Studium (Zeitschrift)

WISU : Wirtschaftsstudium (Zeitschrift)

Die Erläuterung der Symbole und der Variablen erfolgt direkt im Text oder im Anhang, um die für die ökonomische Theorie sowie die Zeitreihenanalyse jeweils übliche Nomenklatur beibehalten zu können. Eine Vereinheitlichung über alle Kapitel hinweg wird strikt vermieden, da gleiche Symbole und Variablen in diesen Bereichen häufig unterschiedlich belegt sind (Beispiel: $\mathrm{u}=$ Arbeitslosenrate sowie Störvariable). 